\title{
X-RAY BASED IMAGING AND 3D RECONSTRUCTION OF DENTAL PATHOLOGIES ON THE EXAMPLE OF PALAEOANTHROPOLOGICAL FINDING
}

\author{
A.V. Gaboutchian ${ }^{1 *}$, V. A. Knyaz ${ }^{2,3}$, H.Y. Simonyan ${ }^{4}$, G.R. Petrosyan ${ }^{5,6}$, D.V. Korost ${ }^{7}$, M.M. Novikov ${ }^{8}$, A.A. Kudaev ${ }^{7}$, \\ S.A. Cherebylo ${ }^{8}$, N.V. Kharlamova ${ }^{9}$ \\ ${ }^{1}$ Peoples' Friendship University of Russia, 117198, Moscow, Russia - armengaboutchian@mail.ru \\ ${ }^{2}$ State Research Institute of Aviation System (GosNIIAS), 125319 Moscow, Russia - knyaz@gosniias.ru \\ ${ }^{3}$ Moscow Institute of Physics and Technology (MIPT), Dolgoprudny, Russia \\ ${ }^{4}$ Scientific Research Centre of the Historical and Cultural Heritage, Yerevan, Armenia - haksimon@ gmail.com \\ 5 Plekhanov Russian University of Economics Yerevan branch, Armenia \\ ${ }^{6}$ International Scientific-Educational Centre of NAS RA, Yerevan, Armenia - petrosyan_gohar@list.ru \\ ${ }^{7}$ Faculty of Geology, Moscow State University, 119234, Moscow, Russia - dkorost@mail.ru ; a.a.kudaev@gmail.com \\ ${ }^{8}$ Research Center Crystallography and Photonics RAS, Shatura, Russia - novikov@ rambler.ru \\ ${ }^{9}$ Institute of Ethnology and Anthropology RAS, 119991, Moscow, Russia - natasha_kharlamova@iea.ras.ru
}

\section{Commission II, WG II/10}

KEY WORDS: Micro-computed tomography, Cone-beam computed tomography, 3D reconstruction, Palaeopatology, Shengavit, Dental pathology.

\begin{abstract}
:
Findings from Bronze Age burials of Shengavit settlement have become a source of multiple studies referred to anthropological, and especially odontological, research based on $3 \mathrm{~d}$ imaging and image processing techniques. The currently presented case is an example of palaeopathological analysis of bone tissue resorption caused by complications of dental pathologies. Thus by analogy with diagnostic procedures in clinical dentistry, conventional x-ray based cone-beam tomographic scanning have been applied and have shown its effectiveness as a study method. Through CBCT imaging we managed to reveal a hidden pathological process in the body of the studied semi-mandible fragment, though initially another pathological area located on the same finding was planned to be studied. Application of micro-computed tomography has improved analytical, or diagnostic, part of the current palaepathological study. It has brought to finding unusual morphological features hypothetically causing bone resorption as a complication of dental pathological conditions. However our intention to obtain $3 \mathrm{~d}$ reconstructions as evidence supporting the most likely version required several attempts to correct image processing in line with the increase of imaging resolution.
\end{abstract}

\section{INTRODUCTION}

Skeletal remains play a significant part in archaeological and anthropological research. Craniology and odontology, which are based on studies of skull and teeth, are usually of particular interest as the mentioned structures and their morphological analysis provide essential information for historical, biological, ethnical, cultural, dietary and other interpretations. At the time studies of dental pathology are the meeting point for both anthropology and dentistry, supported by corresponding imaging and reconstructive techniques, among which x-ray based methods have taken at a certain moment the leading role $(\mathrm{Wu}$ and Schepartz, 2009; Zaim et al., 2011).

This has happened largely due to their ability to reveal hidden structures, to study their physical parameters, morphology and other characteristics. For instance cephalometric and other studies are carried out by means of cone-beam tomographic reconstructions as well as conventional planar x-rays (Lisboa et al., 2015; Afify et al., 2019), providing reliable reconstruction accuracy and results in measurements in many cases (Sam et al., 2019, Gaboutchian and Knyaz, 2019).

However studies of teeth due to their high density and small size usually require reconstructions possessing relatively higher resolutions when different imaging techniques are compared

\footnotetext{
* Corresponding author
}

(Maret et al., 2014) and that therefore micro-computed tomography is often applied for reconstruction of teeth in a wide variety of studies, including palaeopathological dental studies of caries lesions (Fuss et al., 2014; Arnaud et al., 2016; Towle et al., 2019). CBCT and micro-CT methods are often intersected in their diagnostic applicability, though each of possesses effective features. Thus the combined use of these two methods has become the part of the current study. The studied sample was scanned by the both methods for obtaining morphological reconstructions of teeth and bone tissue in different resolutions, which provided the basis for detecting bone resorption and analysis of possible causative pathologic conditions.

The study material is represented by mandibular fragment from Shengavit - Bronze Age settlement (town) situated within the boundaries of Yerevan - the capital of the Republic of Armenia. Shengavit occupied the territory of more than sixty thousand square meters. According to some estimates around five thousand people inhabited this area; they manufactured pottery, had well-developed metal industry, agriculture, livestock farming and winemaking. Excavations of Shengavit necropolis have provided essential information for historical, anthropological and archaeological interpretations, previously presented in our studies (Gaboutchian and Knyaz, 2019, Gaboutchian et al., 2020).

The currently studied semi-mandible fragment has been found 
from Tomb Nr. 7 of the Shengavit necropolis. The burial contained a number of artefacts as well, which allow dating it back to XXVII-XXV centuries BC.

\section{MATERIAL AND METHODS}

The left mandibular fragment possesses a segment of dental arch of three lower left teeth with fully preserved coronal parts: lateral incisor, canine and first premolar. The first molar is represented by two separately situated roots; the coronal part had been completely lost. The bone tissue around these roots has marked signs of resorption involving periapical and root furcation areas, penetrating buccal cortex of the mandible especially in the projection of the mesial root of the tooth. The mandibular fragment has empty sockets of the second premolar and the second molar which, most likely, have been lost post-mortem.

The mandibular fragment was scanned on conventional, designed for use in clinical dentistry, cone-beam tomographic device (Vatech PaX-i 3D, VATECH CO., Ltd., South Korea) in two different modes: FoV 100x75 and FoV 50x50 in order to study bone around the first molar roots - an area of expected changes in bone tissue of inflammatory origin. Thus the tomographic reconstruction revealed bone resorption around separated roots of the first molar (Figure 1a). The lesion involves two separate areas around the distal root and around the mesial; the latter destroying inter-root septum and cortex of the mandible. In addition, another pathological area, though significantly smaller in size, was located in the projection of the lateral incisor root apex after this x-ray diagnostic imaging procedure. However the tooth did not possess any visible signs of decay or breakage (Figure 1b).

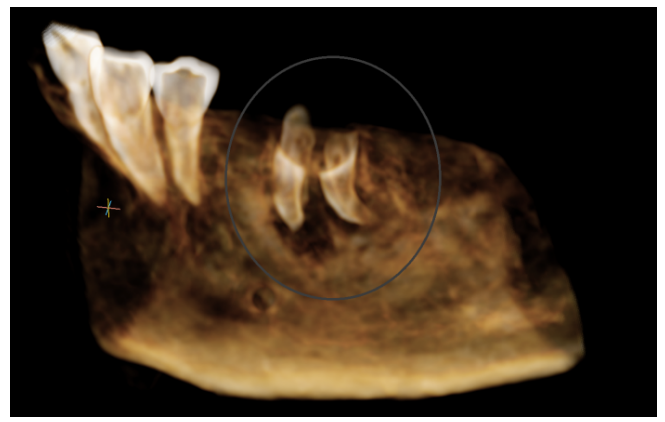

(a)

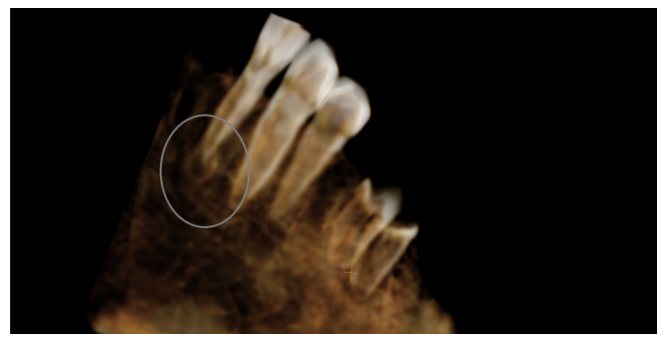

(b)

Figure 1. Areas of bone resorption around the lower left first molar (a) and lower left second incisor (b) on the CBCT reconstruction, FoV 50x50

The planned dental studies regarding the preserved teeth of the semi-mandible from Shengavit as well as more profound palaeopathological studies required conducting micro-computed tomography as well. Thus the obtained through these high resolution reconstructions data was used for analyses of the pathological areas, including analysis of teeth, as very often bone resorptions are odontogenic (caused by complications of dental pathologies). The procedure was performed on multi-purpose industrial tomography scanner General Electric: Phoenix v-tome-x S240; microfocus x-ray tube was applied providing inter-slice distance of $97.8 \mu \mathrm{m}$ and corresponding to it resolution of $3 \mathrm{~d}$ reconstruction. More than eight hundred images 8-bit depth .bmp format were obtained for reconstruction of the current sample (Figure 2).

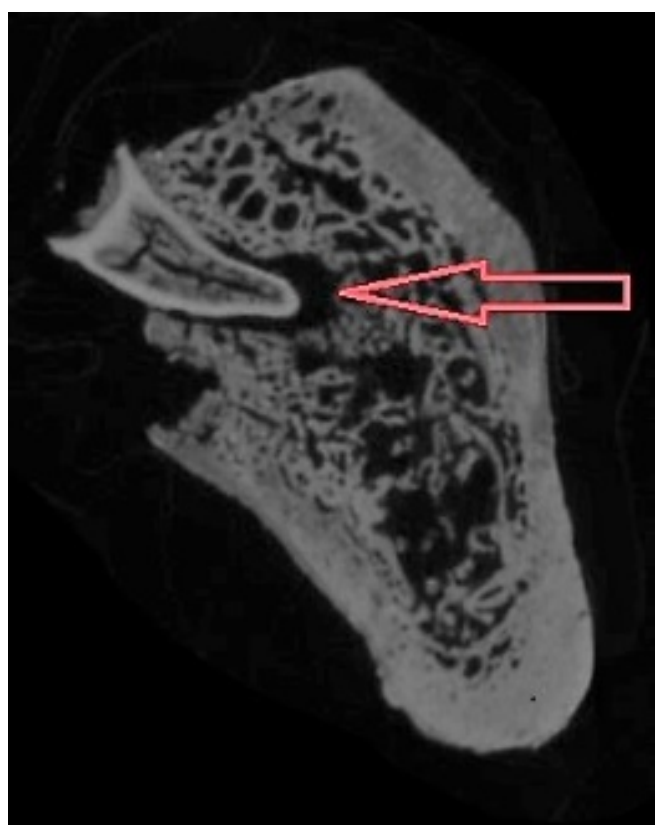

Figure 2. Tomographic slice passing through the distal root of the lower molar. The arrow points at periapical bone resorption area

Reconstructive procedures following imaging provided obtaining detailed $3 \mathrm{~d}$ models of layers corresponding to variety in $\mathrm{x}$-ray absorption by different tissues which compose tooth. Thus the four teeth preserved on the studied semi-mandible were reconstructed according to their enamel, dentin and pulpal chamber (excluding the lower molar with complete absence of coronal part and hence impossibility of enamel reconstruction). Image processing was performed on Avizo software /Amira/ version 9.01; reconstructions were obtained with isometric voxel edge of $97.8 \mu \mathrm{m}$ (Figure 3). 3d image processing procedures were also performed on Magics and Mimics /Materialise/ software.

\section{RESULTS AND DISCUSSION}

The visible bone resorption area around the first molar roots was caused by inflammatory processes which could most likely develop as an implication of complete tooth coronal destruction caused, as an example, by caries. The complete loss of the tooth crown up to complete separation of roots is a hindering factor for analysis of its possible reasons. However we can assume that the initial process could start from the mesial part of the tooth crown and could be accompanied by pulpal chamber deformation providing longer "survival" of distal parts of the tooth. This is due to larger sizes of bone resorption area around the mesial root, which has destroyed the buccal cortex of the mandible - an evidence of multiple inflammation exacerbations throughout the lifetime of the individual. 


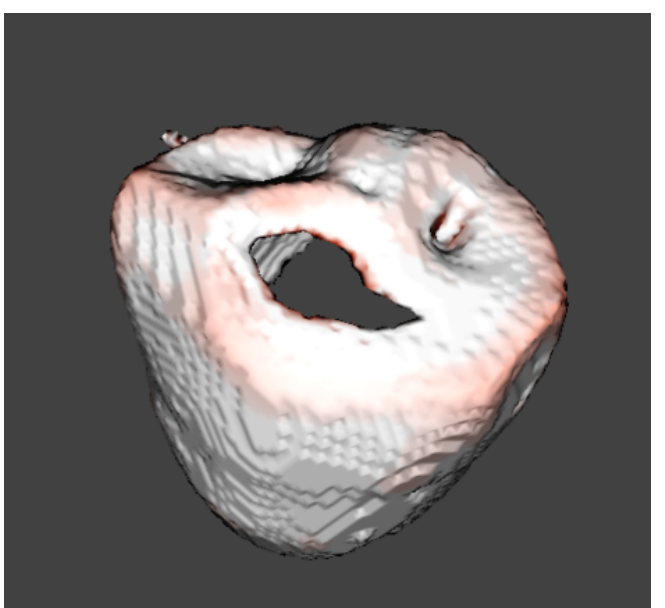

Figure 3. 3d reconstruction of the lower left first premolar enamel cap

Nevertheless this is distal roots with their broader clearance of a single root canal (if compared to two narrower canals of the mesial root) usually serve as easy pathways for penetration of infection through dental tissues and involvement of bone. Hence the distal root apex is surrounded by resorption area - smaller and isolated, not forming fistula.

CBCT has showed its high diagnostic potential as provided for localising a smaller in size pathological process hidden in the depth of the chin portion of the studied mandible and around the apical part of the individuals' incisor root. However the reasons of the local resorption remained unclear as no signs of caries lesions were found during the tooth examination neither on the tomographic reconstruction. Hypothetically we could refer it to possible traumatic injury and subsequent formation of cyst (arguments for this version are partially supported by shallower alveolus of the adjacent, however missing, central incisor).

However studies of high resolution tomographic slices revealed interesting morphological features regarding the incisor. We found connection between slightly attrited (to initial stage of dentin exposure) incisal edge of the tooth and its coronal part of the pulpal chamber on images obtained through micro-CT (Figure 4 a). These changes could be referred to post-mortem influence of factors dissolving dentin, however such an assumption runs counter with the detected periapical bone resorption and absence of caries lesions on the studied incisor. Thus analysis of micro-CT images helped us to reveal correct projections depicting our observations on CBCT reconstructions as well (Figure $4 \mathrm{~b}$ ).

We should mention that similar to pulp horn extensions which occur as unusual dental morphological variation. Thus this can be shown on Figure 5 representing sectioned upper premolar, which pulpal chamber reaches enamel penetrating the entire dentin layer at a certain area of buccal cusp (taken from Khera et al., 1990).

We should mention that pulpal exposure caused by dental wear and resulting periapical bone loss is a common pathology in clinical practice. And thus we have paid attention to attrition level characteristic to the current sample. The obtained $3 \mathrm{~d}$ reconstructions from micro-CT scanner were very informative in terms of attrition evaluation as they demonstrated subtle dentin exposure on the edge of the lateral incisor and cusp tips of the canine and the first premolar. We can assume that new methods

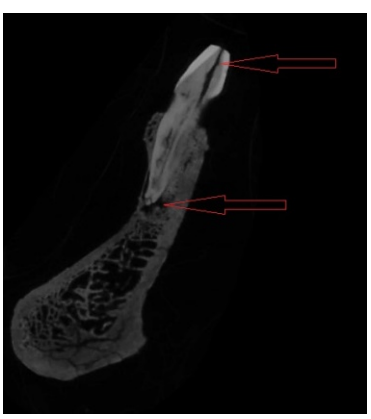

(a)

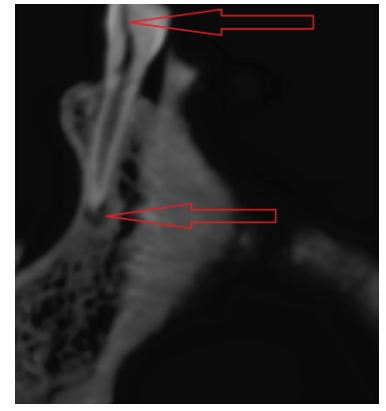

(b)
Figure 4. Micro-tomographic slice (a) and cone-beam tomographic images (b) of the lower left lateral incisor. Arrows point at pulp extension and bone resorption areas

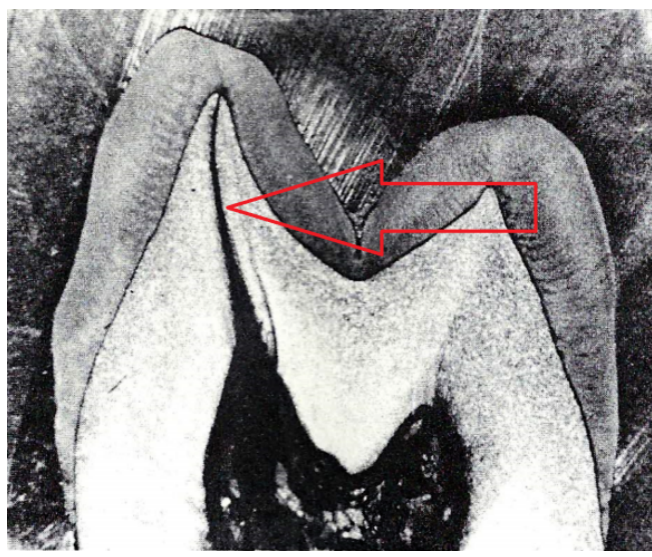

Figure 5. Tooth histological section showing pulp horn extension (Khera et al., 1990)

of dental attrition evaluation can be developed on the bases of such or similarly performed reconstructions. In general, attrition assessments are hindered by incomplete dental semi-arch (only the segment of three teeth in a row is preserved, and molars, which are highly informative, are not preserved).

Nevertheless, according to Alexandr A. Zubov (Zubov, 1968) our observations indicate score 2 on all of the mentioned teeth, and score 2-3 is registered according to Mikhail M. Gerasimov (Gerasimov, 1955), which thereby allows to estimate the age of the individual as approximately around 30 years old. If we refer to other dental wear scoring methods with more diverse graduations of attrition, the individuals' age can be associated with condition corresponding to 20-24 years (phase D according to Lovejoy, 1985). However this means that such a degree of dental wear could barely cause periapical inflammation, and if so only in combination with unusual morphology (similar to shown on Figure 5).

Both imaging techniques provide tomographic sections and $3 \mathrm{~d}$ reconstructions of the observed pathological foci. These reconstructions of the studied mandible, including teeth, were performed with resolutions according to the obtained isometric voxel sizes (Table 1). CBCT reconstructions despite more than 1.5-fold presented difference showed sufficient accuracy in combining reconstructions, which can be clearly observed through reconstruction sectioning (Figure 6). Thus the both modes are applicable for skeletal reconstructions including their pathologies. Nevertheless micro-computed tomography provides higher reconstruction resolution and thus we referred 
to them in order to diagnose the reasons of bone resorption around the incisor root.

\begin{tabular}{|c|c|c|c|}
\hline \multirow[t]{2}{*}{ Reconstruction } & \multicolumn{2}{|c|}{ CBCT } & \multirow[t]{2}{*}{ Micro-CT } \\
\hline & FoV $100 \times 75$ & FoV 50x50 & \\
\hline Voxel size, $\mathrm{mm}$ & 0.32 & 0.2 & 0.1 \\
\hline
\end{tabular}

Table 1. Comparative reconstruction resolutions.

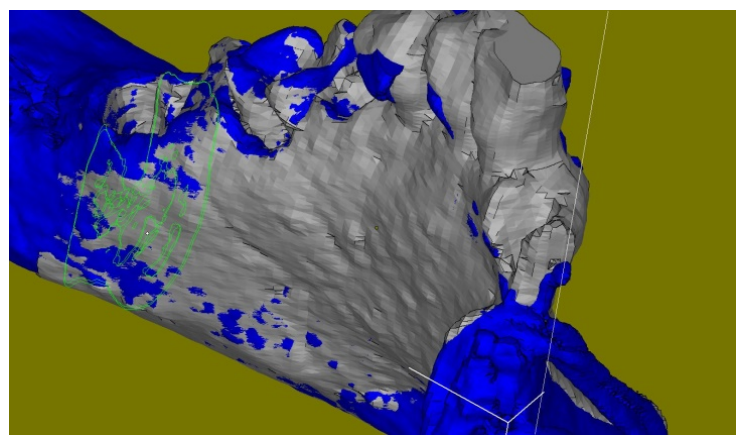

Figure 6. Combining CBCT reconstructions obtained with different fields of view

As it was expected the observations on $2 \mathrm{~d}$ images, hypothetically referred to pulpal cavity extensions, could be reconstructed on $3 \mathrm{~d}$ models. Two different approaches to reconstructions were taken in order to reveal areas of pulpal chamber extension reaching the edges of enamel. A combined $3 \mathrm{~d}$ model of enamel and pulpal chamber was obtained including sectioning (Figure $7 \mathrm{a}$ and $\mathrm{b})$.

Nevertheless none of them depicted the location studied. Presumably this could result from insufficiency of the obtained resolution if compared to the size of the detected morphological feature. However more careful adjustments of thresholding while processing images brought to objective depiction of pulpal chamber on $3 \mathrm{~d}$ reconstructions. We can clearly detect an opening on the incisal edge of the studied tooth providing direct connection of inner structures with destructive environmental influence, including pre- and post-mortem infectioning and erosion. We should mention in addition that the pulpal chamber has an irregular shape, very unusual for those observed on patients requiring endodontic treatment in dental clinical practice.

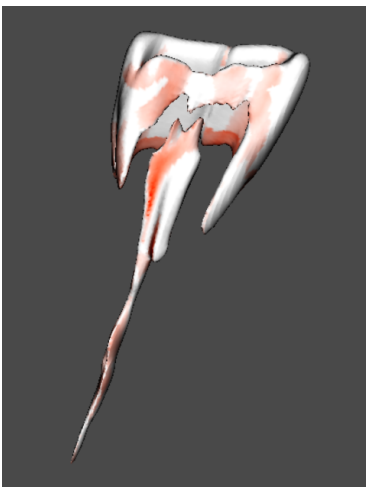

(a)

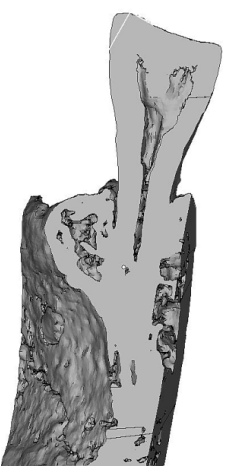

(b)
Figure 7. Reconstructions showing pulpal chamber morphology in combination with enamel $(\mathrm{a}-\mathrm{left})$ and as a tooth section $(\mathrm{b}-$ right)

Thus we can assume that formation of the registered condition is connected with unusual dental morphology which had brought to infectioning of intra-dental soft tissues soon after exposure of dentin caused by normal dental wear (Figure 8 a). Other versions, such as tooth decay, should be carefully examined as well insofar as threshold adjustments show other perforations of root, which, due to their location can barely be caused by caries (Figure $8 \mathrm{~b}$ ). Further extension of the pathological process through the root apical foramen brought to chronic inflammatory process accompanied by formation bone resorption area. Subsequent post-mortem environmental factors provided for formation of irregular cavernous shape of the pulpal chamber (Figure $8 \mathrm{c}$ ) which can be observed on the destroyed roots of the lower first molar as well (Figure 2).

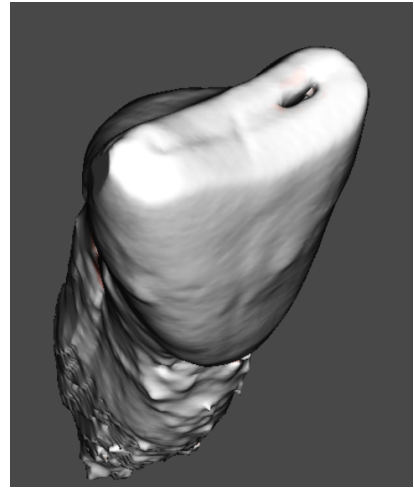

(a)

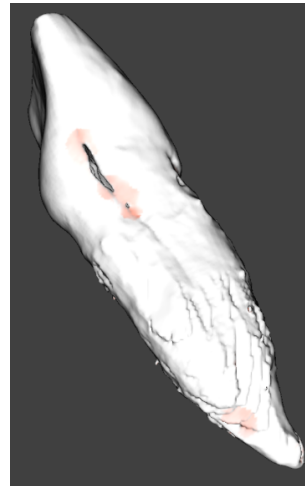

(b)

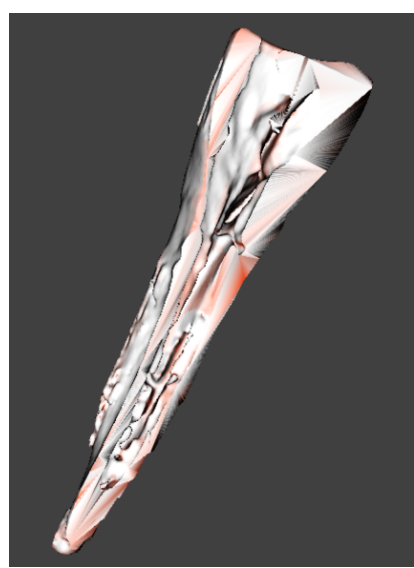

(c)

Figure 8. Reconstructions showing incisal edge opening (a), an opening othe mesial surface of the rooth (b) and cavernous pulpal chamber morphology on a section (c)

Our further studies and diagnostic procedures for odontological (dental anthropological) palaepathological research should be continued in line with new attempts aimed to increasing imaging resolution for providing detailed digital reconstructions. Other methods should be applied as well for more complete studies.

\section{CONCLUSION}

Reconstructive techniques based on $\mathrm{x}$-ray imaging have proved their effectiveness anthropological studies, especially in diagnostics of bone tissue resorptions, either hidden or evident, in palaeopathological research. However more profound diagnostics referred to imaging teeth would require higher resolutions achievable through use of micro-computed tomography 
rather than cone-beam computed tomography. Imaging resolution, as well as image processing, play an important part in diagnostic procedures of palaeopathological research, especially with respect to studies of dental structures.

\section{ACKNOWLEDGEMENTS}

We would like to express our gratitude to Dr H. Avakov for organising the cone-beam tomographic imaging.

\section{REFERENCES}

Afify et al., 2019. Afify, M., Salem, W., Mahmoud, N. Age Estimation from Pulp/Tooth Area Ratio of Canines using ConeBeam Computed Tomography Image Analysis: Study of an Egyptian Sample. 10.4172/2157-7145.1000434

Arnaud et al., 2016. Arnaud, J., Benazzi, S., Romandini, M., Livraghi, A., Panetta, D., Salvadori, P., Volpe, L., Peresani, M. A Neanderthal deciduous human molar with incipient carious infection from the Middle Palaeolithic De Nadale cave, Italy. American Journal of Physical Anthropology. 162. 10.1002/AJPA.23111

Fuss et al., 2014. Fuss, J., Uhlig, G., Böhme, M. Earliest evidence of caries lesion in hominids reveal sugar-rich diet for a Middle Miocene dryopithecine from Europe. PLOS ONE. 13. e0203307. 10.1371/journal.pone.0203307

Gaboutchian and Knyaz, 2019. Gaboutchian, A. V. and Knyaz, V. A.: 3D IMAGES FOR AUTOMATED DIGITAL ODONTOMETRY, Int. Arch. Photogramm. Remote Sens. Spatial Inf. Sci., XLII-2/W18, 53-57, https://doi.org/10.5194/isprsarchives-XLII-2-W18-53-2019, 2019.

Gaboutchian et al., 2019. Gaboutchian, A. V., Knyaz, V. A., Leybova, N. A., Petrosyan, G., Simonyan, H., and Vasilyev, S. V.: APPLICATION OF PHOTOGRAMMETRIC TECHNIQUES IN PALAEOODONTOLOGICAL STUDIES TROUGH AUTOMATED DIGITAL SHAPE ANALYSIS OF HUMAN TEETH, Int. Arch. Photogramm. Remote Sens. Spatial Inf. Sci., XLII-2/W12, 75-80, https://doi.org/10.5194/isprsarchives-XLII-2-W12-75-2019, 2019.

Gaboutchian et al., 2020. Gaboutchian, A. V., Knyaz, V. A., Leybova, N. A., Simonyan, H. Y., Novikov, M. M., Apresyan, S. V., Cherebylo, S. A., and Petrosyan, G. R.: 3d reconstruction and image processing of anthropological archaeological findings, Int. Arch. Photogramm. Remote Sens. Spatial Inf. Sci., XLIII-B2-2020, 845-850, https://doi.org/10.5194/isprsarchives-XLIII-B2-2020-845-2020, 2020

Gerasimov, 1955. Gerasimov M.M. "Vosstanovleniye lica po cherepu” (in Russian). Tr. In-ta etnografiyi AN SSSR, Moscow, 1955, Vol. XXVIII

Khera et al., 1990. Khera S.C., Carpenter C.W., Staley R.N. Anatomy of cusps of posterior teeth and their fracture potential. - JPD, 1990, v 64, 2, p. 139-147.

Lisboa et al., 2015. Lisboa, C., Masterson, D., da Motta, A. F., Motta, A. T. Reliability and reproducibility of threedimensional cephalometric landmarks using CBCT: a systematic review. Journal of applied oral science, 23(2), 112-119. doi:10.1590/1678-775720140336

Lovejoy, 1985. Lovejoy O. Dental Wear in the Libben Population: Its Functional Pattern and Role in the Determination of
Adult Skeletal Age at Death. American journal of physical anthropology. 68. 47-56. 10.1002/ajpa.1330680105

Maret et al., 2014. Maret, D., Peters, O., Galibourg, A., Dumoncel, J., Esclassan, R., Kahn, J.-L., Sixou, M., No, T. Comparison of the Accuracy of 3-dimensional Cone-Beam Computed Tomography and Micro-Computed Tomography Reconstructions by Using Different Voxel Sizes. Journal of Endodontics. 40. 10.1016/j.joen.2014.04.014

Towle et al., 2019. Towle, I., Irish, J., De Groote, I., Fernée, Ch. Dental caries in human evolution: frequency of carious lesions in South African fossil hominins. 10.1101/597385.

$\mathrm{Wu}$ and Schepartz, 2009. Wu X, Schepartz L.A. Application of computed tomography in paleoanthropological research. Progress in Natural Science 19 (2009) 913-921. doi:10.1016/j.pnsc.2008.10.009

Zaim et al., 2011. Zaim, Y., Ciochon, R., Polanski, J., Grine, F., Bettis, A., Rizal, Y.,Franciscus, R., Larick, R., Heizler, M., Aswan, A., Eaves, K., Marsh, H. New 1.5 million-year-old Homo erectus maxilla from Sangiran (Central Java, Indonesia). Journal of human evolution. 61. 363-76. 10.1016/j.jhevol.2011.04.009

Zubov, 1968. Zubov, A.A. "Odontologiya. Metodika antropologicheskikh issledovaniy”(in Russian), 1968, pp 200, Nauka, Moscow 